\title{
GRAEME SEGAL'S BURNSIDE RING CONJECTURE
}

\author{
BY J. FRANK ADAMS
}

1. Introduction. My theme will be that algebraic topology still offers problems which reveal the present state of our art as inadequate. Such problems make us feel that there could be something good going on; but if there is, we have yet to understand it.

I shall devote $\$ 2$ to explaining Segal's original conjecture. In §3-§6 I shall review what is proved about it so far. Broadly, for those few groups we have been able to handle the conjecture is found to be true, and there is no group for which the conjecture is known to be false. In \$7-\$9 I shall explain further conjectures related to the original one; the fact that these also are not yet disproved contributes to the impression that there could be something good going on. In $\$ 10$ I shall comment briefly on our chances of going further.

2. Statement of the conjecture. In this section I shall explain Segal's original conjecture. I must begin by explaining cohomotopy.

Let $X, Y$ be a good pair of spaces, for example, a finite-dimensional $\mathrm{CW}$-pair. Then we have

$$
\pi^{n}(X, Y)=\operatorname{Lim}_{m \rightarrow \infty}\left[S^{m} X / Y, S^{m+n}\right] .
$$

Here maps and homotopies preserve the base-point; and if $Y=\phi$, I use Atiyah's convention that $X / \phi$ means $X$ with a disjoint base-point. We can rewrite the right-hand side as

$$
\operatorname{Lim}_{m \rightarrow \infty}\left[X / Y, \Omega^{m} S^{m+n}\right]
$$

or as

$$
\left[X / Y, \operatorname{Lim}_{m \rightarrow \infty} \Omega^{m} S^{m+n}\right] .
$$

The last expression gives us a definition of $\pi^{n}(X, Y)$ valid whether the pair $X, Y$ is finite dimensional or not.

Cohomotopy is a generalised cohomology theory, namely the one corresponding to the sphere spectrum; and its coefficient groups are the stable homotopy groups of spheres. So it is like stable homotopy; if you could compute it, it would give you a lot of information, but unfortunately it is hard to compute.

However, there is one case in which we have a conjecture, and it is due to Graeme Segal. He asks for the analogue of a well-known theorem of Atiyah [3].

Presented to the Symposium on the Mathematical Heritage of Henri Poincaré, April 7-10, 1980; received by the editors October 28, 1980.

1980 Mathematics Subject Classification. Primary 55Q55, 55R35.

(c) 1982 American Mathematical Societ y 0273-0979/81/0000-0141/\$03.50 
Atiyah wished to compute the $K$-theory of $B G$, the classifying space of $G$, where $G$ was originally a finite group. So Atiyah took the representation ring $R(G)$ and constructed a map

$$
\alpha: R(G) \rightarrow K(B G) .
$$

He then completed $R(G)$ for the topology defined by the powers of the augmentation ideal. He extended $\alpha$ by continuity to get a map

$$
\hat{\alpha: R}(G) \hat{)} \rightarrow K(B G) \text {. }
$$

And finally he proved that this map $\hat{\alpha}$ is an isomorphism.

Segal proposed that we should replace $K$-theory by cohomotopy; so on the right we put $\pi^{0}(B G)$. On the left he proposed to replace the representation ring $R(G)$ by the Burnside ring $A(G)$ of $G$. To define this, instead of taking actions of $G$ on vector spaces, we take actions of $G$ on finite sets. (That is, assuming $G$ is finite, of course.) We divide these actions into isomorphism classes, and make these classes into a semigroup under disjoint union. We take the corresponding Grothendieck group, and that is $A(G)$. We make it a ring so that the product of two finite $G$-sets is their Cartesian product. I emphasise that $A(G)$ should be regarded as something computable and known.

We have again a map

$$
\alpha: A(G) \rightarrow \pi^{0}(B G) .
$$

I will not stop to define it here; the reader may consult $\$ 9$ for a more general construction. We complete $A(G)$ for the topology defined by the powers of the augmentation ideal; and again $\alpha$ extends by continuity to give a map

$$
\hat{\alpha: A}(G) \hat{)} \rightarrow \pi^{0}(B G) .
$$

Segal's conjecture is that this map $\hat{\alpha}$ is an isomorphism.

For the trivial group $G=1$ the conjecture is trivially true; both sides are $Z$ and the map is an isomorphism. This seems to be the only group for which matters are trivial.

3. Results of Lin; the case $G=Z_{2}$. The group $G=Z_{2}$ already presents a

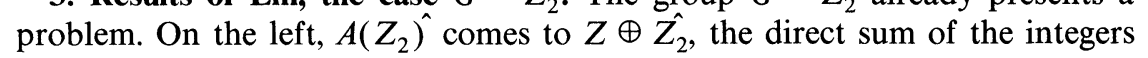
and the 2-adic integers. On the right we can take real projective space $R P^{\infty}$ as our model for $B Z_{2}$, and so the problem is to compute $\pi^{0}\left(R P^{\infty}\right)$; but for ten years nobody could get hold of it. This case was finally solved by W. H. Lin $[11,12]$, using a method which I once heard Graeme Segal call "that damn spectral sequence of yours". Lin found that the conjecture is true for $G=Z_{2}$.

Lin also found that $\pi^{n}\left(R P^{\infty}\right)=0$ for $n>0$. It seems fair to add to our list of conjectures the conjecture

$$
\pi^{n}(B G)=0 \text { for } G \text { finite, } n>0 \text {. }
$$

Let me emphasise that this is not a statement which is true for trivial reasons, in the way that the homotopy groups of a space or spectrum vanish below the Hurewicz dimension. If you try to get hold of $\pi^{n}(B G)$ by obstruction-theory, 
or by an Atiyah-Hirzebruch spectral sequence, you find an infinity of nonzero groups

$$
H^{i}\left(B G ; \pi^{j}(p t)\right) \quad(i+j=n) .
$$

What happens is that these groups all conspire to cancel each other out for $n>0$; for $G=Z_{2}$ this phenomenon was noticed long ago by Mahowald, and later by me [1], although neither of us had a proof.

Various sorts of further argument seem likely to need information about the groups $\pi^{n}\left(R P^{\infty}\right)$ also in the range $n<0$, where they are nontrivial. Lin obtained such information; but to describe the result will require some preliminary explanation.

4. Functional duals. In this section I will explain about functional duals, and so reach the results on $\pi^{n}\left(R P^{\infty}\right)$ for $n<0$.

Let us work in a suitable category of spectra where we can do stable homotopy theory. Consider $\left[W \wedge X, S^{0}\right]$, where $X$ is fixed and $W$ varies. This is a contravariant functor of $W$, and satisfies the hypotheses of Brown's Representability Theorem, so we get a natural (1-1) correspondence

$$
\left[W \wedge X, S^{0}\right] \leftrightarrow[W, D X],
$$

where the representing object $D X$ is by definition the "functional dual" of $X$. In particular,

$$
\pi^{-n}(X)=\left[S^{n} \wedge X, S^{0}\right] \leftrightarrow\left[S^{n}, D X\right]=\pi_{n}(D X) .
$$

So if we know $D X$, we get information about the cohomotopy of $X$.

Let's use the same symbol for a space with base-point and for its suspension spectrum. Then we want to know $D\left(R P^{\infty} / \phi\right)$, and we have

$$
R P^{\infty} / \phi \simeq S^{0} \vee\left(R P^{\infty} / p t\right),
$$

so

$$
D\left(R P^{\infty} / \phi\right) \simeq\left(D S^{0}\right) \vee D\left(R P^{\infty} / p t\right) \simeq S^{0} \vee D\left(R P^{\infty} / p t\right)
$$

Thus it is sufficient to know $D\left(R P^{\infty} / p t\right)$.

Let $M \hat{Z_{2}}$ be a Moore spectrum for the 2-adic integers $\hat{Z_{2}}$ (in dimension 0 ). Let

$$
M \hat{Z_{2}} \wedge\left(R P^{\infty} / p t\right) \rightarrow S^{0}
$$

be any (stable) map for which the functional $S q^{2}$ is nonzero; since the left-hand side is equivalent to $R P^{\infty} / p t$, such maps exist. Let

$$
\left(R P^{\infty} / p t\right) \wedge\left(R P^{\infty} / p t\right) \rightarrow S^{0}
$$

be any map for which the functional $S q^{4}$ is nonzero; again, such maps exist. Then by adjointness we get a map

$$
\left(M \hat{Z_{2}}\right) \vee\left(R P^{\infty} / p t\right) \rightarrow D\left(R P^{\infty} / p t\right) .
$$


Proposition. Any map

$$
\left(M \hat{Z_{2}}\right) \vee\left(R P^{\infty} / p t\right) \rightarrow D\left(R P^{\infty} / p t\right)
$$

constructed in this way is an equivalence.

This is a result of Lin and myself which is not yet written up for publication; I sketch the proof. We have an induced map of homotopy

$$
\pi_{n}\left(\left(M Z_{2}\right) \vee\left(R P^{\infty} / p t\right)\right) \rightarrow \pi_{n}\left(D\left(R P^{\infty} / p t\right)\right) \cong \pi^{-n}\left(R P^{\infty} / p t\right) .
$$

In fact we can obtain an induced map between the Adams spectral sequences which should converge to these groups; we have one spectral sequence converging to

$$
\left[S^{0},\left(M Z_{2}\right) \vee\left(R P^{\infty} / p t\right)\right]_{*}
$$

and one converging to

$$
\left[\left(R P^{\infty} / p t\right), S^{0}\right]_{*} .
$$

It is necessary to insist, however, that this map of spectral sequences carries $E_{r}^{s, t}$ to $E_{r}^{s+1, t+1}$; that is, it raises the filtration degree by 1 . By quoting the algebraic result of [12], it is shown that this comparison map of spectral sequences is an isomorphism.

The reader will notice that the result about the functional dual implicitly contains the result that $\pi^{n}\left(R P^{\infty}\right)=0$ for $n>0$ and the result that $\pi^{0}\left(R P^{\infty}\right)$ is $Z \oplus \hat{Z_{2}}$, as well as information about the groups $\pi^{n}\left(R P^{\infty}\right)$ for $n<0$. Of course, it is still necessary to take the isomorphism $\pi^{0}\left(R P^{\infty}\right) \cong Z \oplus \hat{Z_{2}}$ given by the proposition and reconcile it with the map $\hat{\alpha}$. Similar remarks will apply whenever we describe a functional dual $D(B G)$.

This completes my account of the results for $G=Z_{2}$.

5. Results of Gunawardena and Ravenel; the case $G=Z_{n}$. The next case should be the case $G=Z_{p}$, where $p$ is an odd prime. Here Gunawardena has shown that with suitable care Lin's results go over [9].

The next case should be the case $G=Z_{p^{e}}, p$ prime, $e \geqslant 1$. In this case D. C. Ravenel finds

$$
D\left(B Z_{p^{e}} / p t\right) \simeq\left(\stackrel{e}{\vee} M Z_{p}\right) \vee\left(\underset{i=1}{e} B Z_{p^{i}} / p t\right)
$$

This is in agreement with the conjecture. An announcement of Ravenel's work is in course of publication [14]. The proof sketched lacks only a treatment of the convergence of a spectral sequence constructed for the purposes of the proof; it is plausible that this should not present an essential obstacle. The proof is by induction over $e$, and it relies on the previous work of Lin and Gunawardena to deal with the case $e=1$ and start the induction.

The case of a general cyclic group $Z_{n}$ follows immediately. After all, for a cyclic group $Z_{n}$ we have a stable equivalence

$$
B Z_{n} / p t \simeq \underset{p}{\bigvee} B S_{p} / p t
$$


where $S_{p}$ is the Sylow $p$-subgroup of $Z_{n}$. This gives

$$
D\left(B Z_{n} / p t\right) \simeq \bigvee_{p} D\left(B\left(S_{p} / p t\right)\right)
$$

where the right-hand side is known by the work of Ravenel.

6. Other results. One should expect that suitable statements for a finite group $G$ will follow from the corresponding statements about the Sylow subgroups $S_{p}$ of $G$. For the conjecture " $\alpha$ mono" this was proved by Laitinen [10]. For the conjectures " $\alpha$ iso" and " $\pi^{n}(B G)=0$ for $n>0$ " it has been proved by May and McClure; but I do not have details about publication. In particular, the conjectures should be true for any finite group $G$ all of whose Sylow subgroups are cyclic.

Results which show that $\hat{\alpha}$ is mono are perhaps less impressive than ones which show that $\hat{\alpha}$ is iso. However, Laitinen [10] has proved that $\hat{\alpha}$ is mono when $G$ is an elementary abelian $p$-group. This result was later extended to all finite abelian groups by Segal and Stretch. They use $B P$-theory; again I do not know details about publication.

At this point I more or less run out of positive results to report. Noncyclic groups present a substantial problem. To do calculations (corresponding to those which Lin did for $G=Z_{2}$ ) for so small a group as $G=Z_{2} \times Z_{2}$ seems to be something contemplated only by a select few.

7. Could the functional duals be doing something good? At this point it is natural to step back and see if we can guess some larger pattern into which the pieces might fit. In this section I will do so for the functional duals.

Let $G$ be a finite group, and let $H$ be a subgroup of $G$. Then the coset space $G / H$ is a finite $G$-set. We can also consider the set of $G$-maps $G / H \rightarrow G / H$, and this turns out to be $W_{H}=N_{G}(H) / H$, where $N_{G}(H)$ is the normaliser of $H$ in $G$. So we have $W_{H} \times G$ acting on the finite set $G / H$. Thus we get a finite covering associated to the universal bundle over $B\left(W_{H} \times G\right)$ $\simeq B W_{H} \times B G$, say

$$
G / H \rightarrow E \stackrel{p}{\rightarrow} B W_{H} \times B G
$$

Take the unit element $1 \in \pi^{0}(E)$ and apply the transfer $[2,5,7,8]$; we get an element

$$
\begin{aligned}
p ! 1 & \in \pi^{0}\left(B W_{H} \times B G\right)=\left[\left(B W_{H} \times B G\right) / \phi, S^{0}\right] \\
& =\left[\left(B W_{H} / \phi\right) \wedge(B G / \phi), S^{0}\right] .
\end{aligned}
$$

This corresponds to a map

$$
B W_{H} / \phi \rightarrow D(B G / \phi) .
$$

Conjugate subgroups $H$ lead to the same outcome. So I get a map

$$
\underset{\{H\}}{\bigvee}\left(B W_{H}\right) / \phi \rightarrow D(B G / \phi)
$$


where in the wedge-sum I take one subgroup $H$ from each conjugacy class. The left-hand side contains a summand $S^{0}$ which arises for $H=G$ $\left(W_{G}=N_{G}(G) / G=1\right)$. This maps to the obvious summand $S^{0}$ of $D(B G / \phi)$ (the constant map $G \rightarrow 1$ gives $B G / \phi \rightarrow B 1 / \phi$, which dualises to $D(B 1 / \phi) \rightarrow D(B G / \phi))$. As for the complementary summands, one may conjecture that this map gives an equivalence provided that $G$ is a $p$-group and on the left you complete at the prime $p$. This appears consistent with what is known for cyclic $p$-groups and can be guessed for other groups. If $G$ were not a $p$-group life would become more complicated.

8. Could equivariant cohomotopy be doing something good? In this section I will discuss some generalisations of the original conjecture which involve equivariant cohomotopy.

In a seminar in Oxford in February 1980 I proposed the conjecture in $\$ 7$; and they were very polite and told me that this was indeed the obvious conjecture, and that it could be incorporated into further conjectures which were better and brighter and even more conjectural. These conjectures are again due to Segal, and again he follows an analogy.

Atiyah and Segal long ago generalised Atiyah's theorem

$$
\hat{\alpha}: R(G) \stackrel{\wedge}{\cong} K(B G)
$$

in the following direction [4]. Let $X$ be a suitable $G$-space, for example, a finite $G$ - $C W$-complex in the sense of Matumoto [13]. Then there are two ways to define the equivariant $K$-theory of $X$. First, we can form $E_{G} \times_{G} X$ and take ordinary $K$ of it, so as to get $K\left(E G \times{ }_{G} X\right)$. This way works with $K$ replaced by any generalised cohomology theory $k$; you can form $k\left(E G \times_{G} X\right)$. The second way only works if you have a good geometrical construction for $k$; then you go through the construction again with $G$ acting on everything. In particular, Atiyah and Segal define $K_{G}(X)$ in terms of $G$ vector-bundles over the $G$-space $X$. Then they get

$$
K_{G}(X)^{\wedge} \cong K\left(E G \times_{G} X\right) .
$$

Taking $X$ to be a point, they recover as a special case the theorem

$$
R(G)^{\wedge} \stackrel{\cong}{\rightarrow} K(B G) .
$$

When one comes to replace $K$-theory by cohomotopy, one replaces $K\left(E G \times_{G} X\right)$ on the right by $\pi^{n}\left(E G \times_{G} X\right)$. On the left we should replace $K_{G}(X)$ by the equivariant cohomotopy of $X$ according to some direct definition. For simplicity I will begin with the case $G=Z_{2}$. In this case we have two ways to suspend a given $G$-space: one, say $S$, in which $Z_{2}$ preserves the suspension coordinate, and another, say $T$, in which $Z_{2}$ reverses the suspension coordinate. So let $X, Y$ be a finite-dimensional $G-C W$-pair, where $G=Z_{2}$. We can form

$$
\pi_{G}^{i, j}(X, Y)=\operatorname{Lim}_{m, n \rightarrow \infty}\left[S^{m} T^{n} X / Y, S^{m+i} T^{n+j}\right]_{G} .
$$

Here maps and homotopies are $G$-maps and $G$-homotopies preserving the base-point. Now just as I did in $§ 2$, I can rewrite this in the following form. 


$$
\operatorname{Lim}_{m, n \rightarrow \infty}\left[X / Y, \Omega_{S}^{m} \Omega_{T}^{n} S^{m+i} T^{n+j}\right]_{G}
$$

Here the function-space on the right has the obvious action of $G$; an element $g \in G$ acts on a function $f$ to give $g \circ f \circ g^{-1}$. I can rewrite things again in the following form.

$$
\left[X / Y, \operatorname{Lim}_{m, n \rightarrow \infty} \Omega_{S}^{m} \Omega_{T}^{n} S^{m+i} T^{n+j}\right]_{G} .
$$

In this form the definition is valid whether the pair $X, Y$ is finite-dimensional or not.

For a general finite group $G$ of course I have to allow for suspension using any finite-dimensional real representation of $G$, and I get groups $\pi_{G}^{\rho}(X, Y)$ indexed by elements $\rho$ in the real representation ring $R O(G)$. It is clear enough how the generalisation goes.

On the left of our conjecture we can now put the groups $\pi_{G}^{\rho}(X, Y)$. But on the right we still have ordinary cohomotopy groups

$$
\pi^{n}\left(E G \times_{\dot{G}} X, E G \times_{G} Y\right)
$$

which are indexed over the integers. What is to be done?

It seems the best move is the following. $E G$ is usually considered as a space with $G$ acting on its right, but we can make $G$ act on its left by using $e . g .^{-1}$. Then we can form

$$
\pi_{G}^{\rho}(E G \times X, E G \times Y) .
$$

The constant map $c: E G \rightarrow p t$ of course induces

$$
(c \times 1)^{*}: \pi_{G}^{\rho}(X, Y) \rightarrow \pi_{G}^{\rho}(E G \times X, E G \times Y) .
$$

If $\rho$ happens to be an integer $n$, we can prove

$$
\pi_{G}^{n}(E G \times X, E G \times Y) \cong \pi^{n}\left(E G \times{ }_{G} X, E G \times{ }_{G} Y\right) .
$$

The proof is not quite the same as it was for $K$-theory, but still it can be done. Whether $\rho$ is an integer or not, we can conjecture that $(c \times 1)^{*}$ becomes an isomorphism if we complete the left-hand side. (Notice that $c: E G \rightarrow p t$ may be a homotopy equivalence, but it is not a $G$-homotopy-equivalence.)

On the face of it, it is not clear how the left-hand side $\pi_{G}^{\rho}(X, Y)$ just considered is related to the sort of left-hand side, involving groups $W_{H}$, which we saw in the last section. Experts in this area see a relation, but I am not ready to report on it with confidence.

I believe that when $G$ is cyclic the conjecture in this section can probably be deduced from the results $I$ have described above.

9. Could something make sense for compact Lie groups in general? Up to this point $G$ has been a finite group; I now seek to generalise so that $G$ can be any compact Lie group. Let $(B G)^{m}$ be the $m$-skeleton of $B G$; then we have the following exact sequence due to Milnor.

$$
0 \rightarrow \underset{\leftarrow}{\operatorname{Lim}^{1}} \pi^{*}\left((B G)^{m}\right) \rightarrow \pi^{*}(B G) \rightarrow \underset{\leftarrow}{\operatorname{Lim}^{0}} \pi^{*}\left((B G)^{m}\right) \rightarrow 0
$$


When $G$ is finite the Lim ${ }^{1}$ term is zero, but this need no longer happen when $G$ has positive dimension. It seems advisable to replace $\pi^{n}(B G)$ in our conjecture by $\underset{\leftarrow}{\operatorname{Lim}^{0}} \pi^{n}\left((B G)^{m}\right)$. Once this is done, hope does not seem to be ruled out.

At this point I should give briefly some perspective on the Burnside ring in this context. Take some good class of compact $G$-spaces $X$, for example, finite $G$-CW-complexes. By a $G$-Euler characteristic, I shall mean a pair $(A, \chi)$ of the following sort. $A$ is a given abelian group; $\chi$ is a function which assigns to each finite $G$-CW-complex $X$ an element $\chi(X)$ in $A$; and $\chi$ satisfies the following axioms.

(0) (Zero) $\chi(\phi)=0$.

(i) (Invariance). If $X$ and $Y$ are $G$-homotopy-equivalent, then $\chi(X)=\chi(Y)$.

(iii) (Mayer-Vietoris) Under the obvious assumptions,

$$
\chi\left(X_{1} \cup X_{2}\right)+\chi\left(X_{1} \cap X_{2}\right)=\chi\left(X_{1}\right)+\chi\left(X_{2}\right) .
$$

(Axiom (0) seems to be needed; one can replace $\chi(X)$ by $\chi(X)+c$, where $c$ is a constant, without affecting axioms (i) and (ii).)

Among such $G$-Euler-characteristics, there is one which is universal. In this, the group $A$ is a free abelian group with one generator $a_{\{H\}}$ for each conjugacy class $\{H\}$ of closed subgroups $H \subset G$. The function $\chi$ is defined as follows. Given $X$, let $r(\{H\}, n)$ be the number of $G$-cells in $X$ of type $(G / K) \times E^{n}$ with $K \in\{H\}$; set

$$
\chi(X)=\sum_{\{H\}, n}(-1)^{n} r(\{H\}, n) a_{\{H\}} .
$$

This is the most naive generalisation of the Euler characteristic; we count the $G$-cells with a sign $(-1)^{n}$ depending on their dimension, but we keep accounts separately for $G$-cells of the different symmetry types. For all this, see [6].

We now proceed as follows. For any such $G$-space $X$ we can form the bundle with fibres $X$ associated to the universal $G$-bundle, that is

$$
E G \times{ }_{G} X \stackrel{p}{\rightarrow} B G .
$$

We can take the unit element

$$
1 \in \pi^{0}\left(E G \times_{G} X\right)
$$

and apply transfer $[\mathbf{5}, \mathbf{7}, \mathbf{8}]$ getting an "index"

$$
p, 1 \in \pi^{0}(B G) .
$$

We check that this satisfies the axioms for a $G$-Euler-characteristic of $X$; by the universal property, we get a homomorphism

$$
\alpha: A \rightarrow \pi^{0}(B G) .
$$

(If we use a version of the transfer which is only defined when the base is finite dimensional, or even when the base is a finite complex, we just replace $\pi^{0}(B G)$ by a suitable inverse limit.) 
At this point the general case has one new twist which doesn't show up when $G$ is finite. Let $H$ be a closed subgroup of $G$; then by definition, $\alpha a_{\{H\}}$ is $p,(1)$ in the following fibering.

$$
G / H \rightarrow E G \times_{H} p t \rightarrow B G .
$$

Let $N$ be the normaliser of $H$, and assume that $N / H$ is of positive dimension. Then we can find a 1-parameter subgroup $\theta_{t}$ in $N$ which leads from the identity element to a point not in $H$. Acting on $E G \times_{H} p t$ by right translation with $\theta_{t}$, we can deform the identity map

$$
\text { 1: } E G \times_{H} p t \rightarrow E G \times_{H} p t
$$

to a map without fixed-points. So the fixed-point index $p, 1$ is zero in this case. Thus we can pass to a quotient of our previous universal $A$ in which we map to zero each generator $a_{\{H\}}$ such that $N / H$ is of positive dimension; we leave only the generators $a_{\{H\}}$ such that $N / H$ is finite. We thus reach tom Dieck's version of the Burnside ring $A(G)[6]$, and we still have a homomorphism

$$
\alpha: A(G) \rightarrow \pi^{0}(B G) .
$$

The one case which seems accessible to checking at the present time is the case $G=S^{1}$, for one can approximate to $S^{1}$ by its cyclic subgroups $Z_{n}$. As our model for $B S^{1}$ we can take the complex projective space $C P^{\infty}$. When we audit the conjectures about

$$
\underset{m}{\operatorname{Lim}} \pi^{n}\left(C P^{m}\right)
$$

for $n \geqslant 0$, they seem to stand up.

10. What are our chances? Finally, I owe you some comments about our chances of going further. To begin with, it will be prudent to allow for a chance of say $5 \%$ or $10 \%$ that the conjectures fail when you go beyond cyclic groups. If so, then "calculation is the way to the truth"; one can always hope that a sufficiently heroic effort will settle one more group. On the other hand, if the conjectures are true, then we must ask what sort of a proof to seek. From the attractive nature of some of the formulations, you might be encouraged to hope for a fairly conceptual proof. I don't think it's all that likely, myself. After all, the original formulation was copied from $K$-theory, and in that case the best proof we have is the proof by Atiyah and Segal [4], which works by slowly building up the results for a succession of well-chosen particular groups. It would seem more reasonable to look for a proof like that, and the question is, how to build up.

The first obvious suggestion is to follow the approach used by Atiyah in [3]; try to prove something for $p$-groups $G$ by induction, applying the inductive hypothesis either to a suitable normal subgroup $H \subset G$, or to the corresponding quotient $G / H$, or to both. This is hard and nobody can do it yet; the reader will find that the difficulty is already present in the case $G=Z_{2} \times Z_{2}$.

The second obvious suggestion is to follow the approach used by Atiyah and Segal in [4]; go via compact Lie groups, even if you only want a conclusion about finite groups. This approach also does not seem realistic yet. 
My conclusion is that this area deserves further study. But in order to work in it, those who love conceptual theories had better not scorn calculation, while those who love horrendous calculations should not neglect such help and guidance as may be had from conceptual theory.

\section{REFERENCES}

1. J. F. Adams, Operations of the nth kind in K-theory, and what we don't know about RP ${ }^{\infty}$, London Math. Soc. Lecture Notes No. 11, Cambridge Univ. Press, 1974, pp. 1-9. 4.

2. ___ Infinite loop spaces, Princeton Univ. Press, Princeton, N.J., 1978, especially Chapter

3. M. F. Atiyah, Characters and cohomology of finite groups, Inst. Hautes Études Sci. Publ. Math., No. 9 (1961), 23-64.

4. M. F. Atiyah and G. B. Segal, Equivariant $K$-theory and completion, J. Differential Geometry 3 (1969), 1-18.

5. J. C. Becker and D. Gottlieb, Transfer maps for fibrations and duality, Compositio Math. 33 (1976), 107-133.

6. T. tom Dieck, Transformation groups and representation theory, Lecture Notes in Math., vol. 766, Springer-Verlag, Berlin and New York, 1979.

7. A. Dold, The fixed-point index of fibre-preserving maps, Invent. Math. 25 (1974), 281-297.

8. __ The fixed point transfer of fibre-preserving maps, Math. Z. 148 (1976), 215-244.

9. J. H. C. Gunawardena, Segal's conjecture for cyclic groups of (odd) prime order, J. T. Knight Prize Essay, Cambridge, 1980.

10. E. Laitinen, On the Burnside ring and stable cohomotopy of a finite group, Math. Scand. 44 (1979), 37-72.

11. W. H. Lin, On conjectures of Mahowald, Segal and Sullivan, Math. Proc. Cambridge Philos. Soc. 87 (1980), 449-458.

12. W. H. Lin, D. M. Davis, M. E. Mahowald and J. F. Adams, Calculation of Lin's Ext groups, Math. Proc. Cambridge Philos. Soc. 87 (1980), 459-469.

13. T. Matumoto, On G-CW-complexes and a theorem of J.H.C. Whitehead, J. Fac. Sci. Univ. Tokyo 18 (1971), 363-374.

14. D. C. Ravenel, The Segal conjecture for cyclic groups, Bull. London Math. Soc. 13 (1981), $42-44$.

Pure Mathematics Department, University of Cambridge, Cambridge, England 